[Agr. Biol. Chem., Vol. 33, No. 7, p. 1060 1073, 1969]

\title{
Metabolism of 3,4-Dimethylphenyl N-Methylcarbamate in White Rats
}

\author{
By Junshi MrYamoto, Kimiko Yamamoto and Tamiyo Matsumoto \\ Agricultural Chemicals Research Department, Osaka Works, \\ Sumitomo Chemical Co., Ltd., Osaka \\ Received January 22, 1969
}

\begin{abstract}
Orally administered 3,4-dimethylphenyl $\mathrm{N}$-methylcarbamate labelled with carbon-14 at 4- $\mathrm{CH}_{3}$ was easily absorbed from the gastrointestinal tract of male Wistar rats and distributed into the tissues. Elimination of the radioactivity was rapid and essentially complete; namely during $48 \mathrm{hr}$ approximately $92 \%$ and $5 \%$ of the total radioactivity were excreted respectively into urine and feces. The content of the intact carbamate compound in the urine was less than $0.5 \%$. Major degradation products were identified as 3-methyl-4-carboxyphenyl $\mathrm{N}$-methylcarbamate, its $\mathrm{N}$-hydroxymethyl analog and its component phenol. Much less amount of direct hydrolysis product of the original carbamate, 3,4-dimethylphenol and its conjugated forms was demonstrated. 3,4-Dimethylphenyl N-methylcarbamate is presumed to undergo biodegradation through oxidative pathways.
\end{abstract}

3,4-Dimethylphenyl N-methylcarbamate (Meobal, Sumitomo Chemical Co.) is an insecticidal compound now widely used for the control of various hoppers on rice plant in Japan. This compound is also effective against insects on tea tree and vegetables and some kinds of cattle ticks. The work was initiated to determine the extent of biodegradation and to clarify its metabolic pathways in mammals. This paper deals with the excretion and metabolism in the rats after the oral administration of the compound.

\section{MATERIALS AND METHODS}

Special chemicals and reagents. Carbon-14 3,4-dimethylphenyl N-methylcarbamate labelled at $4-\mathrm{CH}_{3}$ was synthesized in this laboratory. ${ }^{11}$ The chemical purity of the preparation melting at $78 \sim 78.5^{\circ} \mathrm{C}$ (non-active carbamate, $78 \sim 79^{\circ} \mathrm{C}$ ) was above $99.5 \%$ and no radioactive impurities were detected by thin layer chromatography. Its specific activity was $1.83 \mathrm{mCi} / \mathrm{mmole}$. 3,4-Dimethylphenol labelled with carbon-14 at the same site (ca. $1.4 \mathrm{mCi} / \mathrm{mmole}$ ) was also used. In the

1) M. Hazue, Unpublished. subsequent experiment, the radioactive compounds were diluted with the non-active compounds and used.

The following reference compounds were synthesized. 3-Methyl-4-carboxyphenyl N-methylcarbamate: 3methylphenol was allowed to react with acetyl chloride in benzene in ice bath by dropwise addition of pyridine. The resultant 3-methylphenyl acetate (103 $\sim 104^{\circ} \mathrm{C} / 23 \mathrm{mmHg}$ ) was dissolved in nitrobenzene and was kept overnight at room temperature together with alminum chloride. After removing nitrobenzene by steam distillation, the crude crystal of 2-methyl-4hydroxy acetophenone (I) was obtained, which, after recrystallization, was converted to 3-methyl-4-acetophenyl benzylether (II) by reaction at $100^{\circ} \mathrm{C}$ for $5 \mathrm{hr}$ under nitrogen with benzyl bromide in the presence of potassium carbonate in dimethylformamide. The reaction mixture was poured into water and II was extracted with ether from ammonium sulfate saturated aqueous layer. II, dissolved in dioxane, was treated with bromine under alkaline condition to form 3methyl-4-carboxyphenyl benzylether (III), which was extracted with ether from the reaction mixture under acidic condition. III was catalytically hydrogenated in the presence of palladium-charcoal in methanolacetic acid and and 3-methyl-4-carboxyphenol (IV) obtained was recrystallized from water, $\mathrm{mp} 178 \sim 181^{\circ} \mathrm{C}$. Anal. Found: C, 59.85; H, 5.55. Calcd. for $\mathrm{C}_{8} \mathrm{H}_{8} \mathrm{O}_{3}$. 
$1 / 2 \mathrm{H}_{2} \mathrm{O}^{21}: \mathrm{C}, 59.62 ; \mathrm{H}, 5.63 \%$. Tetrahydrofuran solution of IV was kept overnight at room temperature with methylisocyanate and the trace amount of triethylamine and 3-methyl-4-carboxyphenyl N-methylcarbamate (V) thus obtained was purified by recrystallization from methanol, mp $177.5 \sim 180^{\circ} \mathrm{C}$. Anal. Found: $\mathrm{C}, 57.55 ; \mathrm{H}, 5.17 ; \mathrm{N}, 6.57$. Calcd. for $\mathrm{C}_{10} \mathrm{H}_{11} \mathrm{NO}_{4}: \mathrm{C}, 57.41 ; \mathrm{H}, 5.30 ; \mathrm{N}, 6.70 \%$. 3-Methyl4-hydroxymethylphenyl $\mathrm{N}$-methylcarbamate: $11 \mathrm{l}$ was treated with diazomethane to obtain 3-methyl-4-carboxymethylphenyl benzyl ether, which was catalytically hydrogenated in the presence of palladium-charcoal to 3-methyl-4-carboxymethylphenol (VI). To lithium aluminum hydride suspended in tetrahydrofuran was added dropwise tetrahydrofuran solution of VI and the mixture was heated at $60 \sim 63^{\circ} \mathrm{C}$ for $2 \mathrm{hr}$, then kept stand overnight at room temperature. The mixture was added to $5 \%$ of icecold hydrochloric acid, and 3-methyl-4-hydroxymethylphenol (VII) was extracted with ether. Recrystallized from benzene, VII melted at $122 \sim 123^{\circ} \mathrm{C}$. VII was allowed to react with methylisocyanate as described above to convert to 3-methyl-4-hydroxymethylphenyl N-methylcarbamate (VIII), which after recrystallization from a mixture of acetone and petroleum ether, melted at $109 \sim 110^{\circ} \mathrm{C}$. Anal. Found: C, 62.06; H, 6.73; N, 7.06. Calcd. for $\mathrm{C}_{10} \mathrm{H}_{13} \mathrm{NO}_{3}: \mathrm{C}, 61.53 ; \mathrm{H}, 6.71 ; \mathrm{N}, 7.18 \%$. Infra red spectra in nujol of IV, V, VIII and NMR spectra of V, VIII are reproduced in Figs. 5a, 5c, 5e and 6a, 6c, respectively. 3,4-Dimethylphenyl sulfate was prepared by reaction of the phenol with chlorosulfonic acid ${ }^{3}$ and was recrystallized from $80 \%$ aqueous ethanol.

Nicotine adenine dinucleotide, oxidized and reduced (NAD, NADH) and nicotine adenine dinucleotide phosphate, oxidized and reduced (NADP, NADPH) and glucose-6-phosphate used in in vitro experiments were purchased from Sigma Chemical Co. and glucose6-phosphate dehydrogenase, from Boeringer and Soehne.

Administration of 3,4 -dimethylphenyl $N$-methylcarbamate
to rats and collection of samples. Male Wistar rats
weighing from $180 \sim 200 \mathrm{~g}$ were used throughout the
experiment. Oral administration was carried out as
follows; $50 \mathrm{mg} / \mathrm{kg}$ of radioactive 3,4 -dimethylphenyl
$\mathrm{N}$-methylcarbamate $\left(\mathrm{LD}_{50}\right.$ to male rats, $380 \mathrm{mg} / \mathrm{kg}$ )
and $50 \mathrm{mg}$ of gum Arabic were well pulverized and

2) F. Tiemann and C. Schotten, Ber., 11, 778 (1878).

3) J. E. M. Whitehead, A. R. Morrison and L. Young, Biochem. J., 51, 585 (1952). suspended in $5 \mathrm{ml}$ of distilled water. Five $\mathrm{ml}$ of the suspension per $\mathrm{kg}$ of body weight was orally administered through the stomach tube. The animals thus treated were housed in a metabolism cage and urine and feces were collected every $12 \mathrm{hr}$ and stored frozen. Expired radioactive carbon dioxide was trapped, as described elsewhere. 4 Radioactive 3,4-dimethylphenol was dissolved in polyethylene glycol 400 and $4 \mathrm{ml} / \mathrm{kg}$ body weight of the solution $(35 \mathrm{mg} / \mathrm{kg})$ was given orally to male rats. When tissues of the animals were required, the animals were sacrificed by decaptation, immediately immersed in crushed ice and the tissues were dissected out as promptly as possible. After washed with $0.9 \%$ of sodium chloride solution, each tissue was stored frozen in dry ice until analyzed. Blood was obtained by cardiac puncture.

Preparation of samples for radioactivity assay. Blood was hemolyzed by addition of four time volume of distilled water. Each tissue was homogenized with four time weight of distilled water in a Waring blender under cooling. After the $\mathrm{pH}$ had been adjusted to 7.0 , these homogenates and hemolyzed blood were shaken with chloroform three times and chloroform layers were separated and dried over anhydrous sodium sulfate. 3,4-Dimethylphenyl N-methylcarbamate and its component phenol were completely transferred to the chloroform layer. Remaining aqueous layer was processed as described already.4! Feces were macerated with five times of distilled water and an aliquot was heated in $3 \mathrm{~N}$ sodium hydroxide at $100^{\circ} \mathrm{C}$ for $1 \mathrm{hr}$ in a sealed tube. Pooled urine at $\mathrm{pH} 7.0$ was extracted with chloroform. After separation of chloroform layer, aqueous layer acidified with one-tenth volume of in hydrochloric acid, was shaken three times with twice its volume of peroxide free ether and ether layers were combined. The remaining aqueous layer was partially saturated with solid ammonium sulfate $(5 \mathrm{~g} / 10 \mathrm{ml})$, and was shaken with the mixture of peroxide free ether and ethanol (2:1) twice. Each organic solvent layer was dehydrated over anhydrous sodium sulfate. An aliquot of the sample solution prepared as above was pipetted into the counting vial, and radioactivity (disintegration per minute) was measured by a liquid scintillation counter. Scintillator solution composed of $4.8 \mathrm{~g}$ of 2,5-diphenyloxazole, $0.12 \mathrm{~g}$ of 2,2'-p-phenylene-bis-(5phenyloxazole), in 1.2 liters of toluene and 0.8 liters

4) J. Miyamoto, Y. Sato, K. Yamamoto, M. Endo and S. Suzuki, Agr. Biol. Chem., 32, 628 (1968). 
of absolute ethanol was used.

Separation and identification of radioactive metabolites from 3,4-dimethylphenyl N-methylcarbamate. Carbon-14 metabolites in ether extract of urine were separated and purified by silica gel column chromatography. Approximately $75 \mathrm{~g}$ of silica gel (Mallinckrodt, 100 mesh) and $15 \mathrm{~g}$ of Hyflo Supercel were slurred in $n$ hexane and ether $(2: 1)$ and packed to yield a $3 \times 25$ $\mathrm{cm}$ column. The ether extract was evaporated to dryness under reduced pressure in the cold onto about $5 \mathrm{~g}$ of silica gel, which was then mounted on top of the packed column. Elution was carried out with the following solvent sequence; $500 \mathrm{ml}$ of 2 to 1 peroxide free ether- $n$-hexane, $600 \mathrm{ml}$ of peroxide free ether and finally $500 \mathrm{ml}$ of 2 to 1 peroxide free ethermethanol. Each $15 \mathrm{ml}$ fraction was collected at a flow rate of approximately $2 \mathrm{ml} / \mathrm{min}$. Carbon- 14 in eluted fractions was measured with a liquid scintillation counter.

Ether-ethanol extract of urine was evaporated in vacuo at $40 \sim 43^{\circ} \mathrm{C}$ and the residue was washed with ethanol and the precipitate was discarded. DEAEcellulose column chromatography was conducted to separate radioactive metabolites in the ethanol layer. Twenty $\mathrm{g}$ of DEAE-cellulose (Serva, $0.53 \mathrm{meq} / \mathrm{g}$ ), preconditioned with $1 / 100 \mathrm{M}$ of phosphate buffer, $\mathrm{pH}$ 7.0 , was mixed with $20 \mathrm{~g}$ of Hyflo Supercel, thoroughly washed with ethanol and packed to make a $3 \times 30 \mathrm{~cm}$ column. The sample solution was poured onto the column. The radioactive compounds were eluted successively with the following solvent system; 1,300 $\mathrm{ml}$ of ethanol, 2, ethanol to ethanol: distilled water (2:1), each $250 \mathrm{ml}$, gradient concentration, 3. ethanol: distilled water (2:1) to ethanol: $1 / 50 \mathrm{M}$ basic disodium phosphate $(2: 1)$, each $300 \mathrm{ml}$, gradient concentration, 4. ethanol: $1 / 50 \mathrm{M}$ basic disodium phosphate (2:1), $300 \mathrm{ml}$. Each $10 \mathrm{ml}$ of the column eluates was collected at the flow rate of approximately $2.5 \mathrm{ml} / \mathrm{min}$ and its radioactivity was determined.

The ether soluble and ether-ethanol soluble metabolites similarly obtained from the urine of rats given radioactive 3,4-dimethylphenol orally were separated in the similar manner. In every chromatographic separation recovery of carbon-14 from the column was substantially complete.

Radioactive compounds in the eluted peaks obtained from the column chromatography as above as well as those in chloroform extract of the urine and the tissues were further resolved by thin layer chromatography. Silica gel HF (Merck), thickness $0.5 \mathrm{~mm}$, was utilized and radioactive spots developed were detected by a radio thin layer scanner. Radioactivity in each spot was quantitatively determined by scraping the silica gel from the plate, putting it into the counting vial and by measuring its radioactivity. The following solvent systems were used for the development; $n$ hexane : ether:formic acid $(2: 1: 0.1)$ (solvent $A$ ), benzene : acetonitrile $(19: 1)(B)$, benzene : acetonitrile $(3: 1)(C)$, benzene : ethylacetate : acetic acid $(10: 5: 1)$ (D), chloroform : methanol (8:1) (E), ether: methanol $(4: 1)(F)$, benzene: methanol $(4: 1)(G)$, benzene: methylene chloride: methanol $(2: 2: 1)(\mathrm{H})$, chloroform : methanol : conc. ammonium hydroxide $(4: 4: 1)$ (I), chloroform: methanol : conc. ammonium hydroxide $(6: 4: 1)$ (J), chloroform : ethanol : conc. ammonium hydroxide $(1: 1: 0.1)(\mathrm{K})$, chloroform : methanol : acetic acid $(75: 20: 5)(\mathrm{L})$, toluene : ethylformate : formic acid $(60: 40: 3)(\mathrm{M})$, benzene : ethylacetate $(2: 1)(\mathrm{N})$ and $n$-butanol : acetic acid: water $(4: 1: 2)(\mathrm{O})$. The following chromogenic reagents were used besides ultra violet absorption and radioactivity, to detect developed spots; $1 \%$ potassium ferricyanide solution was sprayed on the plate, followed by spraying $1 \%$ ferric chloride ethanolic solution (phenolic reagent) and occasionally aqueous ethanolic solution of 2,4-dinitrophenylhydrazine sulfate (carbonyl reagent) was used.

For the determination of $\mathrm{N}-\mathrm{CH}_{2} \mathrm{OH}$ group in some radioactive metabolites an aliquot of the hydrolyzate by $1 \mathrm{~N}$ hydrochloric acid at $100^{\circ} \mathrm{G}$ for $60 \mathrm{~min}$ was caused to react with Nash reagent, ${ }^{51}$ warmed at $58^{\circ} \mathrm{C}$ for $8 \mathrm{~min}$. Yellow color developed was measured at the wavelength of $412 \mathrm{~m} \mu$.

Hydrolysis of urinary glucuronides by purified calf liver $\beta$-glucuronidase and colorimetric determination of glucuronic acid were accomplished with the procedures described elsewhere. ${ }^{4}$

Transformation of 3,4-dimethylphenyl $N$-methylcarbamate by rat liver enzyme preparations in vitro. Male Wister rats abstained overnight from food were sacrificed by decaptation, and liver was dissected out and washed with $0.9 \%$ of sodium chloride solution. The liver was homogenized with nine time volume of $0.25 \mathrm{M}$ of sucrose in a Potter-Elvehjem homogenizer with a Teflon pestle under cooling. The differential centrifugation of the liver homogenate was carried out with a refrigerated centrifuge $\left(0 \sim 3^{\circ} \mathrm{C}\right)$ as in the following, and various enzyme activities were determined to

5) T. Nash, Biochem. J., 55, 416 (1953). 
characterize the separated fractions. ${ }^{6}$ Ten percent homogenate was centrifuged at $900 \mathrm{~g}$ for $6 \mathrm{~min}$. Precipitate was rehomogenized in $0.25 \mathrm{M}$ sucrose, centrifuged again (nucleus and cell debris). The combined supernatants were span at $5000 \mathrm{~g}$ for $6 \mathrm{~min}$. The precipitate was resuspended in the sucrose solution, and centrifuged again at $5000 \mathrm{~g}$ for $6 \mathrm{~min}$ to obtain mitochondrial fraction. The supernatant and the washing were span at $8000 \mathrm{~g}$ for $13 \mathrm{~min}$ and the pellet was resuspended and recentrifuged (lysosome). Microsomal fraction was sedimented from the combined supernatants through centrifugation at $140,000 \mathrm{~g}$ for $90 \mathrm{~min}$. Each particulate fraction was resuspended in $0.25 \mathrm{M}$ sucrose. The tissue fractions were mixed with the amounts of 3,4-dimethylphenyl N-methylcarbamate or its 'hydroxymethyl' analog and cofactors indicated in the Tables in the final volume of 2 or 3 $\mathrm{ml}$ and shaken at $37.5^{\circ} \mathrm{C}$ for the specified intervals in air. After incubation the mixture was diluted to $6 \mathrm{ml}$ with dsitilled water and extracted three times with twice volume of peroxide free ether and ether layers were obtained. Aqueous layer was then acidified by addition of $1 \mathrm{ml}$ of $10 \%$ of perchloric acid, extracted twice with $15 \mathrm{ml}$ of peroxide free ether. Radioactive compounds in the layers were separated by silica gel thin layer chromatography with the appropriate solvent systems shown above.

\section{RESULTS}

Carbon-14 labelled 3,4-dimethylphenyl Nmethylcarbamate was administered orally to male Wistar rats at the rate of $50 \mathrm{mg} / \mathrm{kg}$ body weight and absorption from the intestinal tract and distribution into the tissues were examined. Only slight symptoms of intoxication began appearing a few minutes after treatment, which lasted approximately half an hour and then disappeared. At 7.5, 15, 30,60, 120 and $240 \mathrm{~min}$ after administration, each three head of rats were sacrificed and content of 3,4dimethyl $\mathrm{N}$-methylcarbamate as well as total carbon-14 in brain, liver, kidney and blood was determined. Figure 1 shows the results. The concentration of total carbon-14 and the carbamate reached the maximum already 15

6) J. Miyamoto, Unpublished.
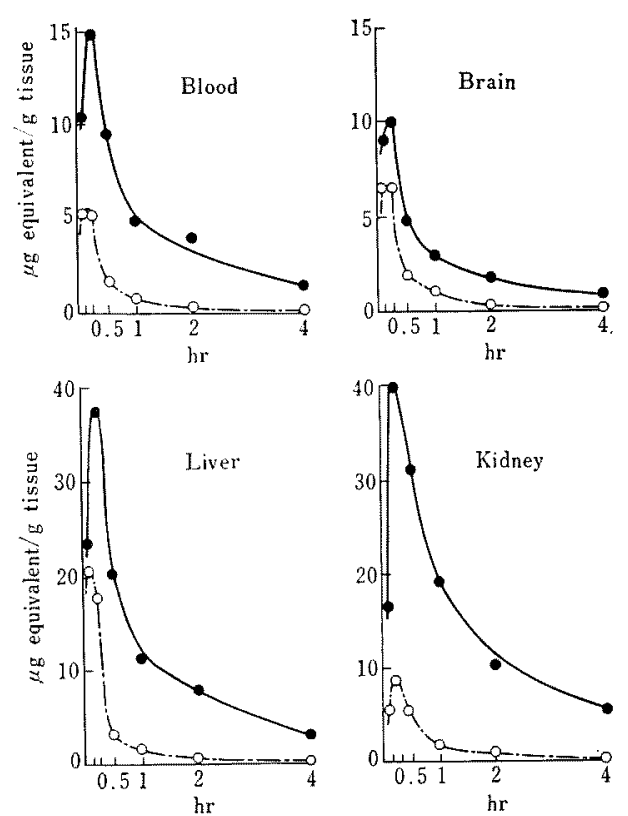

FIG. 1. Content of Total Carbon-14 and 3,4-Dimethylphenyl N-Methylcarbamate in Several Tissues and Blood after Oral Administration of the Radioactive Carbamate Compound.

- Total carbon-14

O 3,4-Dimethylphenyl N-methylcarbamate

min after administration, but it decreased rapidly thereafter and after $4 \mathrm{hr}$ only negligible amount of the original carbamate was present in these tissues and blood. Liver and kidney were found to contain radioactive metabolites other than the carbamate. Excretion of carbon14 was tested after $50 \mathrm{mg} / \mathrm{kg}$ of the carbamate compound had been given orally. The results reproduced in Fig. 2 are the mean value of three replicated trials, using 2 head each of the animals. These results revealed that the radioactivity was rapidly excreted, mainly into urine; namely during $48 \mathrm{hr}$ after administration of the compound $91.5 \%$ and $4.9 \%$ of the radiocativity initially applied were excreted respectively into urine and feces, and during subsequent 3 days only little amount of carbon- 


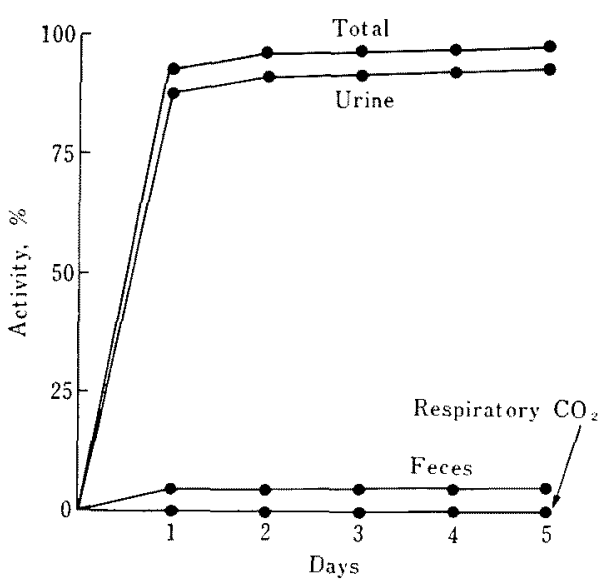

FIG. 2. Cumulative Excretion of Carbon-14 after Oral Administration of Radioactive 3,4-Dimethylphenyl $\mathrm{N}$-methylcarbamate to Male Rats.

14 was further excreted. Thus, more than $97 \%$ of the radioactivity was recovered in 5 day period. Negligible amount of radioactive carbon dioxide was expired, i.e., less than $0.1 \%$ of the total administered during these period of time.

Radioactive metabolites in $48 \mathrm{hr}$ pooled urine obtained from 50 head of rats given orally 50 $\mathrm{mg} / \mathrm{kg}$ of the radioactive carbamate compound, were separated and identified. Unless otherwise stated, the content of carbon-14 compounds in each fraction was expressed in terms of percent of total radioactivity in the pooled urine.

Chloroform extract of the urine (ca. 3\%) was chromatographed on thin layer of silica gel. The content of intact 3,4-dimethylphenyl $\mathrm{N}$ methylcarbamate ( $R f 0.37$ with solvent $\mathrm{A}$ and 0.60 with B) was approximately $0.2 \%$. Free 3,4-dimethylphenol extracted into chloroform ( $R f 0.64$ with $A, 0.82$ with B) amounted to $0.6 \%$. Besides these, chloroform extract contained another radioactive metabolite $(2.2 \%)$ having $R f$ value of 0.05 with solvent $A$. This radioactive metabolite gave single peak with either solvent $\mathrm{C}(R f 0.23)$ or $\mathrm{D}(R f 0.42)$ and cochoromatographed with 3-hydroxymethyl-4- methylphenyl $\mathrm{N}$-methylcarbamate present in chloroform extract of $\beta$-glucuronidase hydrolyzate, as described below in detail. As the amount of the former metabolite was not enough, it was mixed with the latter and NMR spectra of the mixture were determined. The spectra obtained were completely identical with those shown in Fig. 6d.

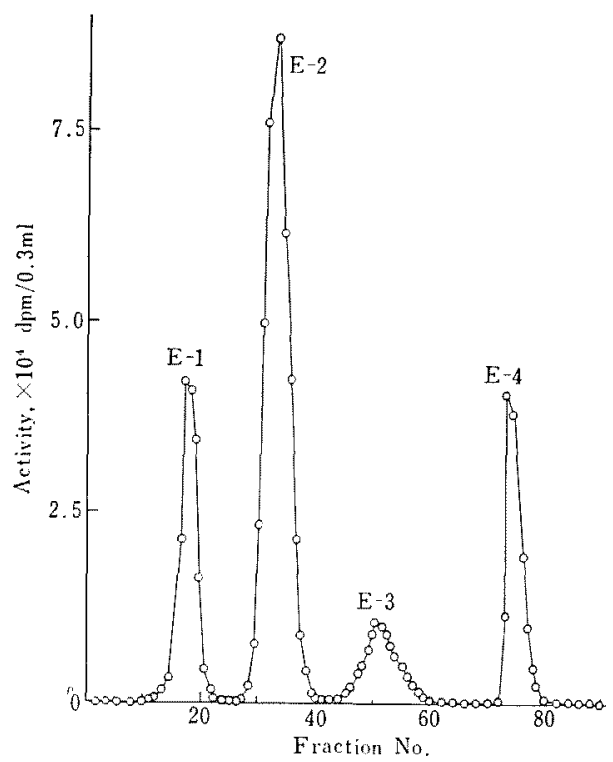

FIG. 3. Silica Gel Column Chromatogram of Carbon14 Metabolites in Acidic Ether Extract of Unine from Rats Dosed 3,4-Dimethylphenyl N-Methylcarbamate.

Acidic ether extract of the urine (55\% of carbon-14) was subjected to silica gel column chromatography, through which radioactive compounds were preliminarily separated into four major peaks. The typical chromatogram is shown in Fig. 3. The structure of some of the radioactive compounds present in these peaks was determined as follows.

Fraction E-1 consisted of one major radioactive compound $(10.1 \%)$ with a trace amount of another one. The former cochromatographed with the authentic 3-methyl-4-carboxy- 
phenol (IV) with solvent A $(R f 0.24)$ or $\mathrm{G}(R f$ 0.22). Recrystallized from water it melted at $177 \sim 179^{\circ} \mathrm{C}$, and its elementary composition after exhaustive drying on phosphorus pentoxide was $\mathrm{C}, 63.07 \%, \mathrm{H}, 5.21 \%$, which coincided well with the formula of $\mathrm{C}_{8} \mathrm{H}_{8} \mathrm{O}_{3}(\mathrm{C}$, $63.15 \%, \mathrm{H}, 5.30 \%$ ). Its IR spectra are reproduced in Fig. 5b, which agrees well with those of IV (Fig. 5a).

Fraction E-2 was found to comprise single radioactive compound cochromatographing with synthetic 3-methyl-4-carboxyphenyl Nmethylcarbamate $(\mathrm{V})$ on silica gel thin layer (solvent $\mathrm{D}, \mathrm{F}$ or $\mathrm{J}$ ). The purified metabolite melted at $178 \sim 181^{\circ} \mathrm{C}$. Anal. Found: C, 57.31; $\mathrm{H}, 5.23 ; \mathrm{N}, 6.41 \%$. The IR and NMR spectra of the compound are reproduced in Figs. 5d and $6 \mathrm{~b}$, which are identical with those of $\mathrm{V}$ (Figs. 5c and 6a). (Throughout the present experiment NMR spectra were measured in 6-deutero-dimethylsulfoxide, using TMS as internal standard. Signals at $\delta, 2.53 \mathrm{ppm}$ originate from dimethylsulfoxide and those in Figs. $6 c$ to $6 \mathrm{e}$ at $\delta, 3.38 \mathrm{ppm}$, from $\mathrm{H}_{2} \mathrm{O}$ contaminated.) Furthermore, the radioactive phenolic compound derived from the metabolite by alkaline hydrolysis ( $1 \mathrm{~N}$ sodium hydroxide at $100^{\circ} \mathrm{C}$ for $30 \mathrm{~min}$ ) cochromatographed with IV Based on these evidences, the metabolite was identified as 3-methyl-4-carboxyphenyl N- methylcarbamate. The content in the urine amounted to $30.1 \%$.

Among two radioactive metabolites in Fraction E-3 $(6.1 \%)$, the major one $(4.9 \%)$ was purified by thin layer chromatography (solvent $\mathrm{M}, R f 0.21$ ). It gave single spot when developed with other solvent systems. On acidic hydrolysis, it produced $96 \%$ of the theoretical amount of formaldehyde, calculated on the basis of radioactivity, and by hydrolysis in $1_{\mathrm{N}}$ sodium hydroxide at $100^{\circ} \mathrm{C}$ for $30 \mathrm{~min}$ it gave rise to radioactive phenol identical with IV by thin layer chromatography. From the above, the structure of the metabolite was presumed to be 3-methyl-4-carboxyphenyl $\mathrm{N}$ hydroxymethylcarbamate.

Fraction E-4 (8.6\%) was found to comprise four radioactive compounds from the results of thin layer chromatographic separation with solvent $F, G$ or $H$, but neither of which was predominant. None have been identified.

When $35 \mathrm{mg} / \mathrm{kg}$ body weight of radioactive 3,4-dimethylphenol was orally administered to male Wistar rats, approximately $93.5 \%$ ot carbon-14 was excreted into urine during 24 hr. Urinary carbon-14 was similarly fractionated. The content of 3,4-dimethylphenol was $5.8 \%$ of the total radioactivity in the urine and carbon-14 was transferred only reluctantly to ether from the acidified urine; by three

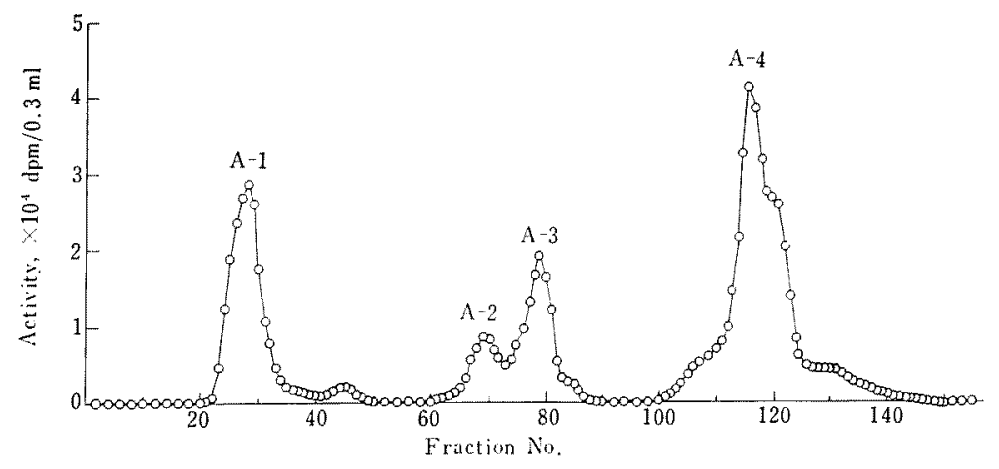

FIG. 4. DEAE-Cellulose Column Chromatogram of Carbon-14 Metabolites in Acidic Ether-Ethanol Extract of Urine from Rats Dosed 3,4-Dimethylphenyl N-Methylcarbamate. 
replicated extractions with twice volume each of ether, ca. $23 \%$ of carbon-14 was partitioned. The ether soluble radioactive metabolites were chromatographed on silica gel column. Carbon-14 was not eluted from the column by ether, but by the mixture of ether and methanol at the fraction No. corresponding to E-4 in Fig. 3. However, none of the ether soluble metabolites from 3,4-dimethylphenol were found identical with those in $\mathbf{E}-4$ by thin layer chromatography with $\mathrm{J}, \mathrm{L}$ or $\mathrm{O}$. Therefore, it seemed that no one of the ether soluble radioactive metabolites from the carbamate compounds (E-1 to E-4) was derived via 3,4dimethylphenol.

Acidic ether-ethanol extract of the urine (40.2\%) was separated by DEAE-cellulose column chromatography into four radioactive peaks, as shown in Fig. 4. The radioactive compounds in Fraction A-I $(9.8 \%)$ were further resolved into four components by thin layer chromatography with solvent $\mathrm{D}$, and those in Fraction A-2 (2.8\%), into two, with L. But neither of them has been characterized. Three carbon-14 metabolites were found in Fraction A-3 (6.1\%), and major one of which (3.5\%) cochromatographed with the authentic sample of 3,4-dimethylphenyl sulfate by thin layer chromatography (Rf 0.36 with L). Hydrolysis of the metabolite in $1 \mathrm{~N}$ hydrochloric acid at $100^{\circ} \mathrm{C}$ for $10 \mathrm{~min}$ yielded a radioactive phenol chromatographically identical with 3,4-dimethyl sulfate.

It appeared that Fraction A-4 (21.5\%) consisted of so many radioactive compounds, as they were hardly separated by thin layer chromatography with solvent $\mathrm{I}, \mathrm{J}, \mathrm{K}$ or $\mathrm{O}$, As this fraction contained bound glucuronic acid, it was subjected to hydrolysis by partially purified calf liver $\beta$-glucuronidase. Hydrolyzate at $\mathrm{pH} 7.0$ was shaken with chloroform, and then with ether under acidic condition, and finally with ether-ethanol, similarly to the original urine. In chloroform extract a minute amount of 3,4-dimethylphenol $(0.8 \%)$ was present. Another radioactive metabolite $(8.20 \%)$ was purified by chromatography on silica gel thin layer using successively with solvent $A$, $\mathrm{C}$ and then with $\mathrm{D}$. This radioactive meta-

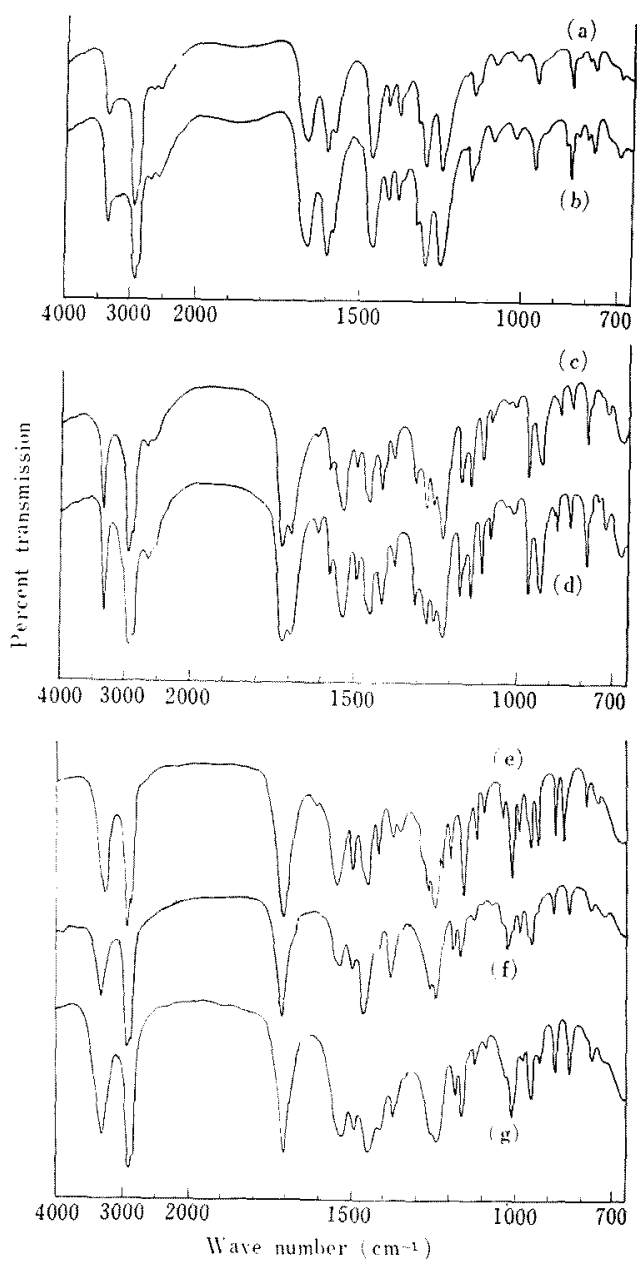

FIG. 5. Infra Red Spectra of Some Metabolites of 3,4-Dimethylphenyl N-Methyl Carbamate.

3-Methyl-4-carboxyphenol, (a) synthesized, (b) obtained from urine. 3-Methyl-4-carboxyphenyl $\mathrm{N}$-methylcarbamate, (c) synthesized, (d) obtained from urine. (e) 3-Methyl-4-hydroxymethylphenyl $\mathrm{N}$-methylcarbamate synthesized, (f) 3-Hydroxymethyl-4-methylphenyl N-methylcarbamate obtained from urine. (g) 'Hydroxymethylphenyl carbamate' formed in vitro. 

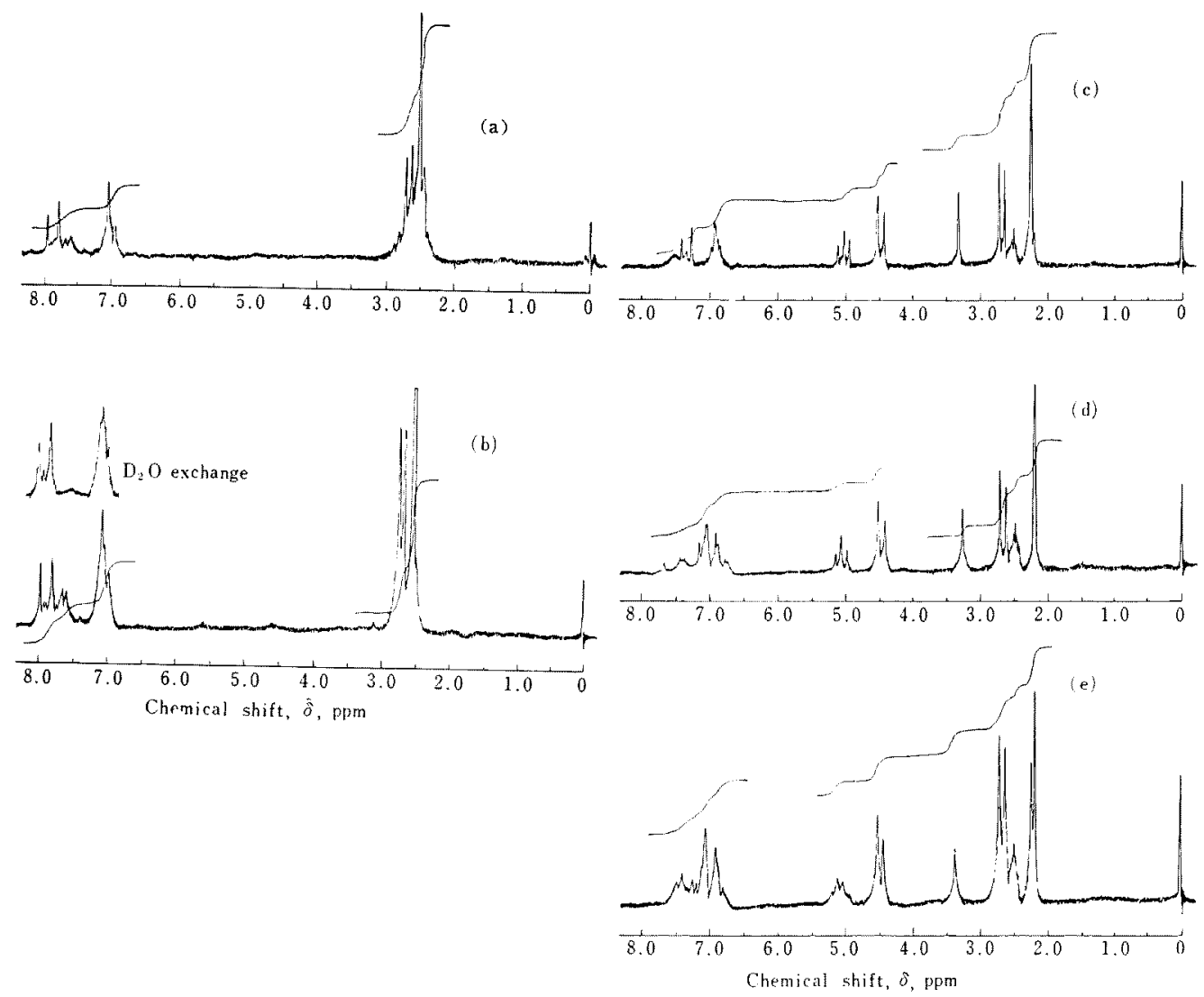

FIG. 6. Nuclear Magnetic Resonance Spectra of Some Metabolites from 3,4-Dimethylphenyl N-Methylcarbamate.

3-Methyl-4-carboxyphenyl N-methylcarbamate, (a) synthesized, (b) obtained from urine. (c) 3Methyl-4-hydroxymethylphenyl N-methyl carbamate synthesized. (d) 3-Hydroxymethyl-4-methylphenyl N-methyl carbamate obtained from urine. (e) 'Hydroxymethylphenyl carbamate' formed in vitro.

bolite was inseparable from synthetic 3-methyl4-hydroxymethylphenyl $\mathrm{N}$-methylcarbamate (VIII) when chromatographed with A, C, D or $\mathrm{E}$, and the radioactive phenol derived therefrom by alkaline hydrolysis cochromatographed with synthetic 3-methyl-4-hydroxymethylphenol with A, C, M or N. However, IR spectra of these two compounds were different as reproduced in Figs. 5e and 5f, and as shown below, NMR spectra of the metabolite in Fig. $6 \mathrm{~d}$ were not identical with those of VIII in
Fig. 6c.

Chemical shift,
$\delta$, ppm. intensity Assigned to Synthetic 3-methyl-4-hydroxymethylphenyl N-methylcarbamate

2.26 (singlet) $\quad 3 \quad \mathrm{CH}_{3}$ attached to

2.67 (doublet) $\quad 3 \quad \mathrm{NH}-\mathrm{CH}_{3}$

4.48 (doublet) $\quad 2 \quad \mathrm{CH}_{2} \mathrm{OH}$

5.02 (triplet) $\quad \mathrm{I} \quad \mathrm{CH}_{2} \mathrm{OH}$

$6.82 \sim 6.97$ (multiplet) 2 aromatic- $\underline{\mathrm{H}}$ 


\begin{tabular}{|c|c|c|}
\hline \multicolumn{2}{|l|}{$7.25 \sim 7.70$ (multiplet, 2} & $\begin{array}{l}\text { aromatic- }-\underline{\mathrm{H}} \text { and } \\
-\mathrm{N} \underline{\mathrm{H}}-\mathrm{CH}_{3}\end{array}$ \\
\hline $\begin{array}{c}\text { broad) } \\
\text { (1) }\end{array}$ & & \\
\hline 2.20 (singlet) & 3 & $\begin{array}{l}\mathrm{CH}_{3} \text { attached to } \\
\text { benzene ring }\end{array}$ \\
\hline 2.67 (doublet) & 3 & $\mathrm{NH}-\mathrm{CH}_{3}$ \\
\hline 4.48 (doublet) & 2 & $\mathrm{CH}_{2} \mathrm{OH}$ \\
\hline 5.08 (triplet) & 1 & $\mathrm{CH}_{2} \mathrm{OH}$ \\
\hline $6.73 \sim 7.17$ (multiplet) & 3 & aromatic- $\underline{H}$ \\
\hline $\begin{array}{c}7.20 \sim 7.60 \text { (multiplet, } \\
\text { broad) }\end{array}$ & & $\mathrm{NH}-\mathrm{CH}_{3}$ \\
\hline
\end{tabular}

From the above considerations, the chloroform soluble metabolite was tentatively identified as 3-hydroxymethyl-4-methylphenyl N-methylcarbamate, rather than VIII, although the authentic sample was not available. In the original urine, therefore, the carbamate metabolite was considered to be present as glucuronide conjugate.

Ether extract of $\beta$-glucuronidase hydrolyzate contained the trace amount of 3-methyl-4carboxyphenol and another radioactive phenol $(4.0 \%)$ having the same $R f$ value as that of the authentic 3-methyl-4-hydroxymethylphenol by thin layer chromatography. However, 3methyl-4-hydroxymethylphenol and 3-hydroxymethyl-4-methylphenol were inseparable with the solvent systems used as mentioned already, the radioactive phenol obtained might be the mixture of them. At least four radioactive spots were detected on developing the acidic ether-ethanol extract of $\beta$-glucuronidase hydrolyzate on silica gel plate. (less than $8 \%$ ). None of them exceeded $3 \%$ in amount and no further attempt was made to determine the structure.

The acidic ether-ethanol soluble metabolites from 3,4-dimethylphenol in the urine were similarly separated into three fractions corresponding to A-1, A-3 and A-4 in Fig. 4. The amounts were respectively 6,9 and $55 \%$ of the total carbon-14 in the unine. The first radioactive peak was resolved into two by thin layer chromatography with the same $R f$ value as that of two of the metabolites in A-1. The second peak solely consisted of 3,4-dimethylphenyl sulfate. The last peak contained three radioactive compounds, among which the major one was 3,4-dimethylphenyl glucuronide. Identity of two other minor components with the metabolites in A-4 was not ascertained.

As the above results in vivo might indicate that 3,4-dimethylphenyl N-methylcarbamate is metabolized in rats mainly through oxidation of methyl group(s) attached to the benzene ring, oxidative degradation of the carbamate compound was tested using rat liver enzyme preparations in vitro. The radioactive carbamate was incubated aerobically with the various liver preparations in the presence of NADPH-generating systems. After incubation at $37.5^{\circ} \mathrm{C}$ for $1.5 \mathrm{hr}$ the reaction mixture was extracted with ether at $\mathrm{pH} \mathrm{7.2,} \mathrm{then} \mathrm{after}$ acidified with perchloric acid. Most of the radioactivity was transferred to ether at $\mathrm{pH}$ 7.2 and the remaining one in aqueous layer was completely extractable into ether under acidic condition. Carbon-14 compounds in these ether extracts was separated by thin layer chromatography. In neutral ether extract two major radioactive compounds were detected with solvent $\mathrm{D}(R f 0.62$ and 0.41 ), one of which with higher $R f$ value was the intact carbamate compound. Another one was inseparable from synthetic 3-methyl-4-hydroxymethylphenyl N-methylcarbamate with solvent C, D or E. This metabolite was referred to 'hydroxymethylphenyl carbamate' Besides these, several minor radioactive spots were detected. The results of degradation of 3,4dimethylphenyl $\mathrm{N}$-methylcarbamate and formation of 'hydroxymethylphenyl carbamate' by liver preparations are indicated in Table I. It is clear from the results that the lysosome-free supernatant (after centrifugation at $8000 \mathrm{~g}$ for $13 \mathrm{~min}$ ) was most active in metabolizing the carbamate compound added, followed by microsomal fraction, and other fractions possessed only low enzyme activity. Kidney homogenate has negligible activity. The major degradation product was 'hydroxy. methylphenyl carbamate' in every case.

Coenzyme requirement in transforming 3,4 
TABLE I. DEGRADATION OF 3,4-DMMETHYLPHENYL N-METHYLCARBAMATE AND FORMATION OF 'HYDROXYMETHYLPHENYL CARBAMATE' by Rat Liver Preparations in vitro

Degradation of Formation of Preparation 3,4-dimethylphenyl 'bydroxymethyl$\mathrm{N}$-methylcarbamate phenyl carbamate'

Liver homogenate $48.1 \%$

5.0 $40.5 \%$ nucleus

2.6

4.7

mitochondria

0.4

2.3

lysosome

lysosome-free supernatant

microsomes

54.8

0.0

40.8

47.5

microsome-free supernatant

Kidney homogenate
35.5

0.8

2.7
TABle II. Coenzyme Requirement For the FORMATION OF' 'HYDROXYMETHYLPHENYL CARBamate' BY RAT LIVER Microsomal Fraction

Degradation of Formation of Coenzyme 3,4-dimethylphenyl 'hydroxymethyl$\mathrm{N}$-methylcarbamate phenyl carbamate'

$\begin{array}{lcc}\text { No coenzyme } & 0.3 \% & 0.2 \% \\ \text { NAD plus NADP } & 2.3 & 1.1 \\ \quad \text { (each } 6 \mu \text { moles) } & & \\ \text { NADH }(6 \mu \text { moles) } & 9.1 & 7.1 \\ \text { NADPH }(6 \mu \text { moles }) & 34.3 & 28.8 \\ \text { NADP }(6 \mu \text { moles), } & 65.7 & 58.6 \\ \text { glucose-6-phosphate }(6 \mu \text { moles }) & \text { plus glucose-6-phos- } \\ \text { phate dehydrogenase }(3.5 \mathrm{IU}) & \end{array}$

The results were the mean value of three replicated trials, and expressed as percent of radioactive carbamate initially added. The incubation mixture contained in $3 \mathrm{ml} 6 \mu$ moles of 3,4-dimethylphenyl $\mathrm{N}$ methylcarbamate, $30 \mu$ moles of niacin, $15 \mu$ moles of magnesium chloride, $100 \mu$ moles of Tris buffer, $\mathrm{pH}$ 7.2 , coenzyme at the specified concentration and $0.4 \mathrm{~g}$ equivalent of rat liver microsomal fraction. Incubated at $37.5^{\circ} \mathrm{C}$ for $1 \mathrm{hr}$ in air.

carbamate' was separated from $150 \mathrm{ml}$ of incubation mixture using microsome fraction fortified with NADPH-generating system and purified by thin layer chromatography. The carbamate thus obtained gave single radioactive spot when developed with every solvent system tested and it was eluted as single peak when chromatographed on silica gel column. However, its IR spectra, reproduced in Fig. $5 \mathrm{~g}$, agreed well neither with those of synthetic 3-methyl-4-hydroxymethylphenyl N-methylcarbamate nor with those of 3-hydroxymethyl4-methylphenyl $\mathrm{N}$-methylcarbamate obtained from urine (Figs. 5e and 5f). The NMR spectra of the metabolite, shown in Fig. 6e, appeared as if they were composite of those of the above two carbamates (Figs. $6 \mathrm{c}$ and $6 \mathrm{~d}$ ) as mentioned below. systems used. The "hydroxymethylphenyl

Synthesized

Assigned to,

aromatic- $\mathrm{CH}_{3}$

aromatic- $\underline{\mathrm{H}}$ and $\mathrm{NH}-\mathrm{CH}_{3}$
$6.82 \sim 7.70(\mathrm{~m})$
From urine

Obtained in vitro

Chemical shift, $\delta, \mathrm{ppm}$
$2.20(\mathrm{~s})$
$2.20,2.26$
$5.08(\mathrm{t})$
$5.07(\mathrm{~m})$

$6.73 \sim 7.60(\mathrm{~m})$

$6.73 \sim 7.70(\mathrm{~m})$ 
The metabolite was, therefore, the mixture of 3-hydroxymethyl-4-methylphenyl- and 3methyl-4-hydroxymethylphenyl- N-methylcarbamate, whose ratio was roughly 3 to 2 if calculated with peak height at 2.20 and 2.26 ppm. 3-Methyl-4-carboxyphenyl N-methylcarbamate (V), if any, was not extracted at $\mathrm{pH} 7.2$, but was extractable into ether under acidic condition. In acidic ether extract of the incubation mixture using microsome, however, only negligible amount of $\mathrm{V}$ was present (about $1 \%$ of the radioactivity initially added). According to the preliminary experiment using liver homogenate fortified with NAD, approximately $10 \%$ of the 'hydroxymethylphenyl carbamate' was converted to $\mathrm{V}$ as shown in Table III, based on the results of thin layer chromatographic separation. Among the minor radioactive metabolites in the incubation mixture, two were positive to

TABLE III. Conversion OF 'HyDROXYMETHYLPHENYL CaRbaMate' to CarboXy ANalog BY RAT LIVER PREPARATION in vitro

Preparation

Liver homogenate "

,

,

"

mitochondria

lysosome-free supernatant microsome

microsome-free supernatant

microsome-free supernatant plus mitocondria

Kidney homogenate
Conzyme

Formation of carboxy analog

Biosynthesized 'hydroxymethylphenyl carbamate' in vitro was used as substrate. Incubation mixture contained $20 \mu$ moles of niacin, $4 \mu$ moles of the coenzyme listed above, $1 \mu$ mole of 'hydroxymethylphenyl carbamate' (mixture of 3-methyl-4-hydroxymethylphenyland 3-hydroxymethyl-4-methylphenyl N-methylcarbamate), $100 \mu$ moles of Tris buffer, $\mathrm{pH} 8.0$ and enzyme preparation, $0.13 \mathrm{~g}$ equivalent of the tissue. Incubated at $37.5^{\circ} \mathrm{C}$, for $1.5 \mathrm{hr}$. The results were mean value of duplicated trials and are expressed as percent of 3-methyl-4-carboxyphenyl $\mathrm{N}$-methylcarbamate formed from the carbamate initially added. carbonyl reagent ( $R f 0.63$ and 0.49 with solvent N), one of which with $R f 0.63$ was also positive to phenolic reagent. This was eluted from the silica gel plate and kept stand with methylisocyanate and triethylamine. As the result its phenol group was covered, still the product remained reactive against carbonyl reagent and was identical in chromatographic behavior with another carbonyl reagent positive metabolite mentioned above. These tndings may indicate that $\mathrm{N}$-methylcarbamate of a phenol carrying aldehyde group was also derived from the 'hydroxymethylphenyl carbamate'

\section{DISCUSSIONS}

Like other N-methyl and N, N-dimethyl carbamate insecticides, 3,4-dimethylphenyl Nmethylcarbamate is an anti-cholinesterase agent, which is characterized by reversible inhibition of the enzyme. Administration of the carbamate chemical to mammals including white rats causes rapid appearance of the signs of intoxication typical of acetylcholine poisoning, and these symptoms disappear fairly rapidly. As reproduced in Fig. 1, oral treatment of white rats with the ring-methyl labelled carbamate compound revealed that the compound was rapidly absorbed from the gastrointestinal tract and distributed into several tissues, and that the compound was eliminated from these tissues and blood also rapidly. The changes in the content were in parallel with the symptoms mentioned above. These tissues contained a considerable amount of radioactivity other than the original carbamate compound, which implies that the compound is easily degraded. Most of the radioactivity given orally to rats was found to be excreted mainly into urine. As shown in Fig. 2 , more than $90 \%$ of the radioactivity initially applied was found in urine and less than $5 \%$ in feces. This seems to be another support of the presumption that the compound was easily absorbed from the intestinal tract. The 
compound was eliminated substantially completely (more than 97\%) from the animal body during 5 day period and there seems to be no retention of radioactivity in the tissues. These results suggest that 3,4-dimethylphenyl $\mathrm{N}$ methylcarbamate has little residual effects on mammals, although possibility of carbamoylation of proteins presumed in the case of some other carbamate compounds ${ }^{7}$ is not always completely excluded. In fact, 90 day feeding study carried out in the laboratory demonstrated that rats tolerated up to $900 \mathrm{ppm}$ of the carbamate chemical with no significant adverse effects other than the slight decrease of cholinesterase activity of plasma and liver. ${ }^{8}$ )

Carbon-14 labelled metabolites obtained after oral administration of $50 \mathrm{mg} / \mathrm{kg}$ body weight of 3,4-dimethylphenyl $\mathrm{N}$-methylcarbamate were separated in $48 \mathrm{hr}$ pooled urine by solvent extraction and by column as well as thin layer chromatography. As the results the urine was found to contain at least 28 radioactive compounds. Among the excreted compounds, unchanged 3,4-dimethylphenyl N-methylcarbamate was found, but its amount was less than $0.5 \%$ of total carbon- 14 in the urine. Although carbaryl (1-naphthyl N-methylcarbamate) or Banol (6-chloro-3,4-dimethylphenyl N-methylcarbamate) were claimed to convert to $\mathrm{N}$ glucuronic acid conjugate in vivo, ${ }^{9,10)}$ the present carbamate compound seemingly did not undergo such conjugation reaction, because bound glucuronic acids in acidic ether-ethanol extract (A-4) were hydrolyzed by $\beta$-glucuronidase nearly to completion (more than 95\%) without liberation of 3,4-dimethylphenyl $\mathrm{N}$ methylcarbamate.

Based on IR and NMR spectra study and cochromatographic examination with the authentic sample, the structure of the major

7) J. G. Krishna and J. E. Casida, J. Agr. Food Chem., 14, 98 (1966).

8) T. Kadota, Unpublished.

9) J. B. Knaak, M.J. Tallant, W.J. Bartley and L.J. Sullivan, J. Agr. Food Chem., 13, 537 (1965).

10) R. L. Baron and J. D. Doherty, ibid., 15, 830 (1967). metabolite in urine was determined to be 3methyl-4-carboxyphenyl $\mathrm{N}$-methylcarbamate (V). N-Hydroxymethyl analog of V presumably derived therefrom was tentatively identified. Their hydrolysis product, 3-methyl-4carboxyphenol, free and conjugated with glucuronic acid, was also demonstrated. The phenol was not detected in the urine of rats given 3,4-dimethylphenol orally, so it might be formed, e.g. from the above oxidized carbamates. The quantity of these four oxidation products was approximately $45 \%$ of carbon-14 in the urine. The presence of 3methyl-4-hydroxymethylphenyl N-methylcarbamate (VIII), a presumed intermediary compound between the original carbamate and $\mathrm{V}$, was examined in the urine. A radioactive compound extracted with chloroform cochromatographed with the authentic sample of VIII with various solvent systems. One radioactive metabolite cochromatographing with VIII was found to be present in chloroform extract of $\beta$-glucuronidase hydrolyzate of A-4 Fraction. However, IR and NMR spectra of this metabolite were not identical with those of VIII, as shown in Figs. 5e, 5f, $6 c$ and $6 \mathrm{~d}$. Based on the studies of these NMR spectra, the structure of the metabolite was presumed to be 3-hydroxymethyl-4-methylphenyl Nmethylcarbamate. The urine contained both free 3-hydroxymethyl-4-methylphenyl Nmethylcarbamate and its glucuronide conjugate. The presence in urine of 3-methyl-4hydroxymethylphenyl N-methylcarbamate has not yet been demonstrated. In ether extract of $\beta$-glucuronidase hydrolyzate a radioactive phenol was present, cochromatographing on silica gel thin layer with synthetic 3-methyl4-hydroxymethylphenol. However, radioactive phenolic compound derived by alkaline hydrolysis of 3-hydroxymethyl-4-methylphenyl $\mathrm{N}$-methylcarbamate also gave the identical $R f$ value with every solvent system used. Thus, it is not certain at present that which one of these two 'hydroxymethylphenols' had been conjugated with glucuronic acid, and it has 
not been clarified either that which one of two $\mathrm{OH}$ groups in such 'hydroxymethylphenol' had been coupled with glucuronic acid. No free 'hydroxymethylphenol' was detected in the urine.

Oxidation of alkyl side chains of substituted $\mathrm{N}$-methylcarbamates is surmised to be catalyzed by liver microsomal enzymes fortified with NADPH in vitro. For example, Hook and Smith $^{11)}$ inferred that liver enzyme preparations oxidized the ring-methyl group of several cresyl N-methylcarbamates and Oonnithan and Casida ${ }^{12)}$ postulated that aromatic methyl group of Banol, Mesurol (4-methylthio-3,5-xylyl $\mathrm{N}$-methylcarbamate), Zectran (4-dimethylamino-3,5-xylyl $\mathrm{N}$-methylcarbamate) and SD 8530 (3,4,5-trimethylphenyl N-methylcarbamate) was oxidized to hydroxymethyl group by liver microsomal fraction in the presence of NADPH. But they also stated that no direct evidences were available. It was observed that when 3,4-dimethylphenyl $\mathrm{N}$-methylcarbamate was incubated with liver microsomal fraction fortified with NADPH or with NADPHgenerating systems, it was transformed to its hydroxymethyl analog, as shown in Table II. The hydroxymethyl analog obtained in vitro was found to be the mixture of 3-methyl-4hydroxymethylphenyl- and 3-hydroxymethyl-4methylphenyl-N-methylcarbamate, based on the compariaon of their NMR spectra as reproduced in Figs. 6c, 6d and 6e, but the former hydroxymethyl analog was not detected in urine. The present authors are inclined to surmise only tentatively that the former once formed would be rapidly oxidized further in animal body to 3-methyl-4-carboxyphenyl Nmethylcarbamate, while since the latter is oxidized only slowly, it was excreted as such and as its glucuronide conjugate.

Further oxidation of such hydroxymethyl group leading ultimately to carboxy analog

11) G.E. R. Hook and J.N. Smith, Biochem. J., 102, 504 (1967).

12) E. S. Oonnithan and J. E. Casida, J. Agr. Food Chem., 16, 28 (1968). formation was not accomplished by liver microsomal oxidizing enzymes in vitro, as in the cases of oxidation of Tolbutamide $(\mathrm{N}-p$ toluenesulfonyl- $\mathrm{N}^{\prime}-n$-butylurea) to $\mathrm{N}$ - $p$-carboxybenzenesulfonyl- $\mathrm{N}^{\prime}-n$-butylurea ${ }^{131}$ and $p$-nitrotoluene to $p$-nitrobenzoic acid, ${ }^{141}$ where liver microsome had catalyzed only partial oxidation to $\mathrm{N}$ - $p$-hydroxymethylbenzenesulfonyl-N'$n$-butylurea and $p$-nitrobenzyl alcohol respectively. Oxidation of $p$-nitrobenzyl alcohol to $p$-nitrobenzoic acid was accomplished by the enzymes present in microsome-free supernatant of rabbit liver. ${ }^{141}$ According to the preliminary experiment shown in Table III, microsomefree supernatant, together with mitochodria or microsomes, of rat liver converted 'hydroxymethylphenyl carbamate' to its carboxy analog to some extent, if it was fortified with NAD. Mitochondria or microsomes were seemingly required to reoxidize NADH to NAD. Although direct evidences are not available, 3-methyl-4hydroxymethylphenyl N-methylcarbamate was presumably oxidized to 3-methyl-4-carboxyphenyl $\mathrm{N}$-methylcarbamate via its aldehyde analog as mentioned above.

3,4-Dimethylphenol formed through hydrolysis of the original carbamate chemical, and its cojugation products, 3,4-dimethylphenyl sulfate and 3,4-dimethylphenyl glucuronide, were also demonstrated in the urine, but the amount of these three metabolites was less than $5 \%$ of total radioactivity in the urine. And relatively little amount of other radioactive metabolites was seemingly identical with those obtained from the urine of rats dosed 3,4-dimethylphenol. These facts might imply that 3,4-dimethylphenyl N-methylcarbamate is rather resistant to hydrolysis in animal body and that biodegradation of the carbamate compound proceeds mainly through oxidative pathways, although 17 radioactive metabolites, amounting approximately to $35 \%$ of the total radioactivity in the urine, have not been

13) J. Tagg, D. M. Yasuda, M. Tanabe and C. Mitoma, Biochem. Pharmacol., 16, 143 (1967).

14) J. R. Gillette, J. Biol. Chem., 234, 139 (1959). 


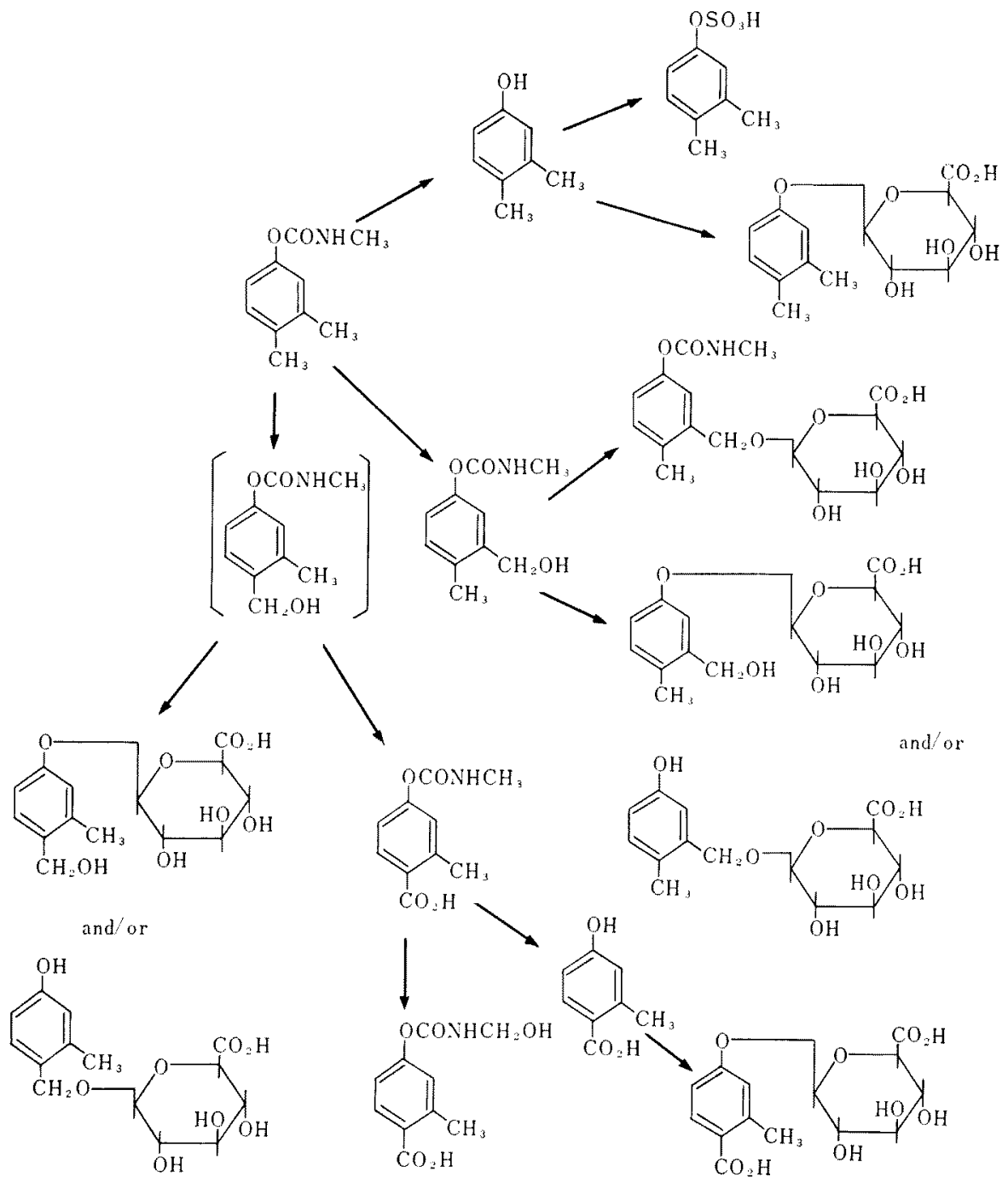

FIG. 7. Proposed Metabolic Pathways of 3,4-Dimethylphenyl N-Methylcarbamate in White Rats.

characterized yet. The metabolic pathways of the carbamate compound in animal body are tentatively proposed, as shown in Fig. 7. Among these metabolites, those having $\mathrm{N}$ methyl or $\mathrm{N}$-hydroxymethyl group in the molecule are supposed to retain biological activity yet. The studies are now in progress on these points and the results will be pub- lished in the following paper.

Acknowledgement. The authors are indebted to Mr. M. Endo, Mr. M. Hazue, Mr. N. Ohmura, Mr. K. Kamoshita, Mr. N. Itaya for their skilled technical assistances and Dr. Y. Nishizawa for his interest and advice. They wish to express their thanks to Sumitomo Chemical Co., Ltd. for permission to publish this work. 